\title{
Percepção de Expressões Faciais Emocionais em Idosos com Doença de Alzheimer
}

\author{
Perception of Emotional Facial Expressions in Alzheimer's Disease
}

\author{
Roberta Ladislau ${ }^{a}$, Josely Gomes Guimarães ${ }^{b}$ \& Wânia Cristina de Souza* $a$ \\ ${ }^{a}$ Universidade de Brasília, Brasília, DF, Brasil \& ${ }^{b}$ Instituto Federal, Brasília, DF, Brasil
}

\begin{abstract}
Resumo
A literatura vem discutindo acerca da percepção de emoções faciais evidentes em idosos com Doença de Alzheimer (DA). Alguns autores sugerem que os déficits apresentados são decorrentes de problemas visuoespaciais; outros sugerem que são por dificuldades no processamento das emoções; e há os que defendem que esse déficit perceptivo é secundário à evolução da demência. Esta pesquisa buscou investigar os aspectos neuropsicológicos da expressão emocional facial, por meio da aplicação da Escala de Inteligência Wechsler para Adultos-III (WAIS-III) e de um programa de computador desenvolvido pelo Laboratório de Psicobiologia denominado Teste de Percepção de Expressões Faciais (TEPEF), que avalia a percepção de faces emocionais em idosos. Os resultados sugerem que o WAIS-III foi sensível para a diferenciação entre os grupos experimental e controle, mas apresentou pouca especificidade. O TEPEF apresentou consistência para avaliar as expressões faciais de alegria, tristeza, nojo, surpresa e raiva. Além disso, ele mostrou que a percepção de alegria em idosos com DA em fase moderada está relativamente preservada. Conclui-se, portanto, que os prejuízos relacionam-se às emoções negativas evidenciadas pela percepção das faces emocionais, demandando supervisão continuada para os idosos com essas alterações.

Palavras-chave: Doença de Alzheimer, face, percepção visual.
\end{abstract}

\begin{abstract}
Literature has been discussing the perception of emotional facial expressions in elderly suffering from Alzheimer's Disease (AD). Some authors suggest that deficits occur due to visuospatial problems; others suggest that the difficulty is related to emotional processing; still others argue that this perceptive deficit is secondary to dementia evolution. This study aimed to investigate the neuropsychological aspects of the perception of facial emotional expression by applying the Wechsler Adult Intelligence Scale-III (WAIS-III) test, as well as a software named Perception Test of Facial Emotional Expressions (TEPEF), which analyzes the perception of emotional faces by the elderly. The results suggest that WAIS-III was sensible in discriminating the differences between experimental and control groups, but presented little specificity. The TEPEF presented consistency to evaluate facial expressions of happiness, sadness, disgust, surprise and anger. Furthermore, it showed that the perception of happiness in the elderly with mild AD is relatively preserved. The expressions more easily perceived - such as happiness and sadness - had correlations with few WAIS-III subtests. Therefore, the study concluded that the losses are related to negative emotions evidenced by the perception of emotional faces, which requires continued supervision for the elderly with these changes.

Keywords: Alzheimer's disease, face, visual perception.
\end{abstract}

Do ponto de vista evolucionista, a face tem importância fundamental para os seres humanos (e para os animais), uma vez que expressa emoções por meio dos movimentos musculares (Darwin, 1872/2000). O seu reconhecimento favorece o comportamento social humano (de Souza,

* Endereço para correspondência: Universidade de Brasília, Instituto de Psicologia, Departamento de Processos Psicológicos Básicos, Campus Universitário Darcy Ribeiro, Instituto Central de Ciências Sul, Brasília, DF, Brasil 70910-900. E-mail: robertaladislau@gmail.com, ajosely@gmail.com e wcdeep@gmail.com

Agradecemos ao apoio do Conselho Nacional de Desenvolvimento Científico e Tecnológico para a pesquisa.
Feitosa, Eifuku, Tamura, \& Ono, 2008; Spikman et al., 2013) e a sua consequente adaptação (Darwin, 1872/2000; Spikman et al., 2013). Dessa forma, ao discriminar as emoções, o indivíduo escolhe a melhor forma de interagir com o outro, promovendo uma comunicação interpessoal eficaz (Hargrave, Maddock, \& Stone, 2002; McLellan, Johnston, Dalrymple-Alford, \& Porter, 2008; Spikman et al., 2013; Weigelt, Koldewyn, \& Kanwisher, 2011).

Uma das estratégias para investigar a percepção emocional é por meio das expressões faciais emocionais. Há evidências de que existem seis expressões emocionais universais: alegria, medo, raiva, nojo, surpresa e tristeza (Darwin, 1872/2000; Ekman \& Friesen, 1971, 1978). As 
principais estruturas relacionadas ao funcionamento de expressões faciais são a amígdala, o córtex pré-frontal medial e o córtex fusiforme (Haxby, Hoffman, \& Gobbini, 2002; McLellan et al., 2008). O processamento emocional tem como substrato neural o sulco temporal superior (STS; de Souza et al., 2008; Haxby et al., 2002), o estriado ventral, o cíngulo anterior e a ínsula. Assim, a ativação do cíngulo anterior e do cíngulo subcaloso está associada à tristeza; a amígdala relaciona-se ao reconhecimento do medo e da alegria (Choleris, Kavaliers, \& Pfaff, 2008; Haxby et al., 2002; Kipps, Duggins, McCusker, \& Calder, 2007; Sato et al., 2002); os gânglios da base e a ínsula favorecem o reconhecimento do nojo (McLellan et al., 2008); o giro fusiforme, o giro temporal superior direito, o giro parahipocampal, o córtex cingulado e o cerebelo relacionam-se à expressão de surpresa (Yoshimura, Kawamura, Masaoka, \& Homma, 2005); o córtex órbito-frontal (Blair \& Cipolotti, 2000), o giro cingulado anterior e a amígdala (Haxby et al., 2002; Lee, Sun, Leung, Chu, \& Keysers, 2013; Sato et al., 2002; Tessitore et al., 2002; Wright, Dickerson, Feczko, Negeira, \& Williams, 2007) são responsáveis pelo processamento da raiva.

No caso da Doença de Alzheimer (DA), estudos (Burnham \& Hogervorst, 2004; Celone et al., 2006; de Souza et al., 2008; Drapeau, Gosselin, Gagnon, Peretz, \& Lorrain, 2009; Guaita et al., 2009; Kohler et al., 2005; Lee et al., 2013; McLellan et al., 2008; Perry et al., 2001; Werheid \& Clare, 2007) mostraram que a amígdala e o lobo temporal do cérebro são afetados, causando prejuízos nas memórias semântica e episódica e no reconhecimento facial e das expressões emocionais da face. Consequentemente, há uma diminuição nas interações sociais, predispondo o idoso com DA a um declínio funcional posterior (Lee et al., 2013).

A DA é uma doença crônico-degenerativa prevalente no envelhecimento, mas que também apresenta formas pré-senis. Acomete, inicialmente, a orientação temporal e as memórias de curto prazo e episódica. Em seguida, ocorrem déficits na linguagem, nos distúrbios de planejamento e nas habilidades visuoespaciais (Caramelli \& Barbosa, 2002), incluindo déficit na percepção de faces emocionais (McLellan et al., 2008).

Fisiologicamente, a DA é caracterizada pela degeneração de células nervosas no cérebro, incluindo o córtex cerebral, o hipocampo, o córtex entorrinal e o estriado ventral, conforme Selkoe (2001, citado por Sereniki \& Vital, 2008), e por modificações na produção de neurotransmissores, como a acetilcolina, que altera as conexões neurais pré-existentes, interferindo, inclusive na percepção visual (Bentley, Driver, \& Dolan, 2008; Farah \& Feinberg, 2005; Forlenza \& Gattaz, 1998; Sereniki \& Vital, 2008).

Considerando essas alterações presentes na DA, foi questionado na literatura se as vias utilizadas para o reconhecimento de faces eram também utilizadas para o reconhecimento de expressões faciais. Contudo, observou-se uma dupla dissociação entre os dois processos perceptivos. Isto é, pacientes que tinham prejuízo no reconhecimento de faces (prosopagnosia; Busigny \& Rossion, 2010; Ramon, Busigny, \& Rossion, 2010) poderiam ter a percepção da expressão das emoções preservadas (Hargrave et al., 2002; Lee et al., 2013; Weigelt et al., 2011).

Idosos com DA podem apresentar déficit no reconhecimento das faces emocionais (Abrisqueta-Gomez, Bueno, Oliveira, \& Bertolucci, 2002; Hargrave et al., 2002; Lee et al., 2013; Luzzi, Piccirilli, \& Provinciali, 2007; Spoletini et al., 2008) e na memória emocional, embora a capacidade de atribuir conteúdo afetivo a fotos de faces emocionais possa permanecer preservada inicialmente (Burnham \& Hogervorst, 2004; Schultz, 2003).

A dificuldade apresentada no reconhecimento de faces emocionais pode estar relacionada à gravidade da DA (Lee et al., 2013), com o comprometimento de estruturas que compõem o sistema responsável pelo processamento das emoções, como a amígdala, córtex temporal anterior e córtex frontal orbital (Grady, Furey, Pietrini, Horwitz, \& Rapoport, 2001; Lavenu \& Pasquier, 2005; McLellan et al., 2008; Wright et al., 2007), especialmente quando o prejuízo é mais acentuado do lado direito do cérebro (Luzzi et al., 2007).

Burnham e Hogervorst (2004) sugerem que o prejuízo na percepção da expressão emocional facial ocorra por um problema visuoespacial ao invés de déficit no processamento das emoções. Farah e Feinberg (2005) afirmam que lesões no córtex visual de associação resultam em déficits visuoespaciais, como a apraxia construcional. Os déficits visuais podem predizer as fases iniciais da DA (O'Brien et al., 2001) e também podem estar envolvidos na alteração da percepção das expressões emocionais.

Assim, a literatura evidencia que a DA está associada a uma dificuldade no reconhecimento de alegria, tristeza, medo e expressões neutras (Kohler et al., 2005). Lee et al. (2013) realizaram um estudo com exames de imagem em um grupo com DA e sugeriram que a alteração na percepção dessas faces é relacionada a uma menor ativação das regiões neurais envolvidas tanto na simulação motora (córtex pré-motor ventral) quanto na emocional (ínsula anterior).

Considerando os estudos citados, o objetivo do presente artigo foi estudar a percepção das expressões emocionais em idosos com DA. Mais especificamente, este estudo buscou investigar aspectos cognitivos da percepção da expressão facial emocional, por meio da aplicação da Escala de Inteligência Wechsler para Adultos-III (WAIS-III; Wechsler, 2004), uma bateria de testes validada e padronizada para a população brasileira.

\section{Método}

A presente pesquisa foi aprovada pelo Comitê de Ética da Faculdade de Saúde da Universidade de Brasília (Registro 141/08). 


\section{Participantes}

Grupo Experimental. Participaram desse grupo 11 idosos diagnosticados com provável DA em fase leve a moderada, sendo dois homens e nove mulheres. A média de idade dos idosos foi de 84 anos $(D P=4,29)$. Em relação à escolaridade, um participante tinha ensino fundamental incompleto, três tinham ensino fundamental completo, quatro haviam concluído o ensino médio, um apenas iniciou o ensino superior e dois concluíram a graduação. Adotou-se como critério de inclusão escores na Escala Clínica de Demência (CDR; Morris, 1997) igual a um ou dois. Um dos participantes, com três anos de escolaridade, apresentou Mini-Exame do Estado Mental (MEEM; Folstein, Folstein, \& McHugh, 1975) menor que dez, mas permanecia com CDR dois e, por isso, foi incluído. No total, sete idosos apresentaram CDR 2 e quatro CDR 1. Oito participantes diagnosticados com DA foram encaminhados pelo Centro de Medicina do Idoso, do Hospital Universitário de Brasília, e três pela Casa do Vovô, uma instituição que abriga idosos. Destes, dois frequentavam a instituição como hospital-dia e um estava institucionalizado devido à DA e à dificuldade de manejo dos familiares com esse participante. $\mathrm{O}$ diagnóstico dos idosos com DA foi feito baseado nos critérios do CID10 (Código Internacional de Doenças). Além disso, os dados foram corroborados pelas informações contidas nos prontuários e pela avaliação neuropsicológica realizada nesta pesquisa.

Grupo Controle. Participaram do Grupo Controle 13 idosos sem prejuízos cognitivos, sendo três homens e dez mulheres. Eles foram recrutados pelo método bola de neve. Portanto, houve a indicação de idosos conhecidos que, por sua vez, indicavam outros. A idade média desse grupo foi de 69,6 anos $(D P=9,57)$. Um participante tinha ensino fundamental incompleto, três haviam concluído o ensino médio, um apenas iniciou o ensino superior e sete concluíram a graduação.

\section{Estímulos}

Foi utilizado o software TEPEF (Teste de Percepção de Expressões Faciais; Ladislau, 2010), desenvolvido no Laboratório de Psicobiologia do Instituto de Psicologia da Universidade de Brasília. O software continha seis fotos de expressões faciais de cada emoção básica (i.e. alegria, tristeza, nojo, medo, raiva e surpresa), totalizando 36 expressões. Cada expressão foi apresentada duas vezes, num total de 72 tentativas. Foi adotada como critério de acerto a escolha correta da expressão nas duas tentativas disponibilizadas, com o intuito de confirmar a opção selecionada e evitar escolhas ao acaso.

Os estímulos contemplavam faces emocionais de jovens adultos, adultos e idosos, tanto do sexo feminino quanto do masculino. A face emocional aparecia no centro da tela e abaixo da foto havia sete botões: seis continham os nomes de cada emoção (e.g. medo, alegria) e o sétimo mostrava a expressão "não sei", caso o idoso não conseguisse classificar a face emocional em nenhuma das emoções listadas. O experimentador clicava no botão correspondente à resposta do participante e a tela mudava automaticamente para outra expressão. A sequência da apresentação das fotos foi semi-randômica, uma vez que duas expressões iguais ou da mesma emoção não poderiam ser apresentadas consecutivamente.

\section{Instrumentos}

Foi utilizada uma bateria de testes para avaliar as habilidades cognitivas dos participantes, especialmente as habilidades visuoespaciais e as verbais. Por isso, utilizou-se o WAIS-III. Os escores da participante idosa, com idade acima de 89 anos, foram calculados baseados na tabela de 85-89 anos do manual. Incluiu-se o MEEM para rastreio de demência, segundo os parâmetros de Brucki et al. (2003) e o CDR para a verificação do estágio da demência no Grupo Experimental. Foram aplicados ainda a Escala de Depressão Geriátrica (EDG) de Yesavage et al. (1982), com 30 itens e o TEPEF.

\section{Procedimento}

A pesquisa foi realizada entre três e quatro sessões e a aplicação foi individual. A primeira sessão foi designada para a assinatura do Termo de Consentimento Livre e Esclarecido e para a anamnese. A segunda e, quando necessária, a terceira sessões foram usadas para a aplicação do WAIS-III, do MEEM e da EDG. A última sessão foi dedicada ao TEPEF e, posteriormente, à devolutiva aos participantes. Quando o idoso demorava a responder, a instrução era repetida e caso ele não conseguisse definir sua resposta em alguma tentativa, marcava-se "não sei", mas isso ocorreu em poucas tentativas. Ao final da sessão, foi mostrado um gráfico gerado pelo TEPEF com o resultado obtido.

\section{Análise Estatística}

Os dados foram analisados em grupo, correlacionando as informações das avaliações com os dados do TEPEF. Para essa análise, utilizou-se o software SPSS 16 (Statistical Package for the Social Sciences). O banco de dados foi submetido aos métodos não paramétricos do teste de correlação de Spearmann e do teste de Kruskal-Wallis devido à amostra pequena $(n=24)$. Esse foi escolhido também por comparar amostras independentes e não pareadas. A hipótese da presente pesquisa é que o Grupo Experimental apresentaria mais dificuldades no reconhecimento das faces emocionais que o Grupo Controle.

\section{Resultados}

Em relação às análises intragrupos dos testes neuropsicológicos aplicados (WAIS-III, MEEM e EDG), observou-se diferenças significativas tanto no WAIS-III (Tabela 1) quanto no MEEM, caracterizando o nível de comprometimento do Grupo Experimental $(p<0,05)$. Contudo, a EDG não apresentou diferença significativa entre os grupos (Tabela 2). 
Ladislau, R., Guimarães, J. G. \& Souza, W. C. (2015). Percepção de Expressões Faciais Emocionais em Idosos com Doença de Alzheimer.

Tabela 1

Teste de Kruskal-Wallis para a Comparação dos Subtestes do WAIS-III entre Grupos, considerando a Significância Estatística

\begin{tabular}{cccc}
\hline & \multicolumn{2}{c}{ Escore equivalente ao posto médio } & \\
\cline { 2 - 3 } Testes & Grupo Experimental & Grupo Controle & Significância $(p)$ \\
\hline Completar Figuras & 7 & 13 & $<0,001$ \\
Vocabulário & 7 & 10 & 0,005 \\
Códigos & 6 & 12 & $<0,001$ \\
Semelhanças & 7 & 11 & $<0,001$ \\
Cubos & 7 & 11 & $<0,001$ \\
Aritmética & 6 & 10 & 0,001 \\
Raciocínio Matricial & 7 & 13 & $<0,001$ \\
Dígitos & 8,59 & 10 & 0,012 \\
Informação & 7 & 9 & 0,001 \\
Arranjo de Figuras & 7 & 11 & $<0,001$ \\
Compreensão & 5 & 11 & $<0,001$ \\
Procurar Símbolos & 8 & 12 & $<0,001$ \\
Sequência Número-Letra & 5 & 10 & $<0,001$ \\
Armar Objeto & 5 & 11 & $<0,001$ \\
\hline
\end{tabular}

Tabela 2

Teste de Kruskal-Wallis para a Comparação dos Índices Fatoriais do WAIS-III, MEEM e EDG entre Grupos, considerando a Significância Estatística

\begin{tabular}{cccc}
\hline \multirow{2}{*}{ Testes } & \multicolumn{2}{c}{ Escore equivalente ao posto médio } & \multirow{2}{*}{ Significância $(p)$} \\
\cline { 2 - 3 } & Grupo Experimental & Grupo Controle & \\
\hline QI Verbal & 82 & 101 & $<0,001$ \\
QI Executivo & 83 & 116 & $<0,001$ \\
QI Total & 79 & 109 & $<0,001$ \\
ICV & 84 & 105 & $<0,001$ \\
IOP & 81 & 115 & $<0,001$ \\
IMO & 83 & 104 & 0,002 \\
IVP & 84 & 111 & $<0,001$ \\
MEEM & 13 & 27 & $<0,001$ \\
EDG & 14 & 4 & 0,161 \\
\hline
\end{tabular}

Notas. ICV = Índice de Compreensão Verbal; IOP = Índice de Organização Perceptual; IMO = Índice de Memória Operacional; IVP = Índice de Velocidade de Processamento; MEEM = Mini-Exame do Estado Mental; EDG = Escala de Depressão Geriátrica. 
Importante notar que não houve pareamento na variável idade $(p<0,001)$. Observou-se que o Grupo Controle foi composto por idosos mais jovens (média de 69,6 anos, com desvio padrão de 9,57) que os do Grupo Experimental (média de 84, com desvio padrão de 4,29). Assim, as análises realizadas ao longo da pesquisa podem sofrer influência do envelhecimento, pois essa variável não foi adequadamente controlada.

Em relação às análises dos testes neuropsicológicos aplicados (WAIS-III, MEEM e EDG), o teste não paramétrico Kruskal-Wallis mostrou diferenças significativas tanto no WAIS-III quanto no MEEM, caracterizando o nível de comprometimento do Grupo Experimental (Tabelas 1 e 2). Comparando os dois grupos, observa-se $p<0,05$ para Vocabulário, Dígitos, IMO e $p \leq 0,001$ para os outros subtestes, índices fatoriais e para o MEEM. Os resultados da EDG não mostraram diferença significativa entre os grupos, embora o posto médio do Grupo Experimental tenha escore indicando depressão leve-moderada e o Grupo Controle tenha posto médio sem indícios de depressão. A EDG foi respondida pelos idosos com DA, sem interferência dos familiares ou cuidadores. É possível que haja viés nesse resultado pela capacidade cognitiva do participante estar alterada.

O desempenho dos idosos dos dois grupos no TEPEF satisfez a condição de acerto para as duas tentativas, com índices em torno de $75 \%$ no Grupo Controle para as faces emocionais de tristeza, nojo, surpresa e raiva. Para a face emocional de alegria, o índice de acerto foi de quase $100 \%$, enquanto que para a face emocional de medo, o reconhecimento foi de $12,82 \%$. O Grupo Experimental, por sua vez, obteve índice de reconhecimento próximo de $80 \%$ para a face de alegria; de $36 \%$ para a de tristeza e as restantes ficaram abaixo de $20 \%$ de reconhecimento, sugerindo que o reconhecimento da face de alegria é a única que está preservada nesse grupo. $\mathrm{O}$ desempenho dos participantes para a percepção de faces diferiu significativamente $(p<0,001)$, exceto para medo $(p=0,186)$, sugerindo que apenas os estímulos de medo não tiveram relação com as condições cognitivas dos participantes avaliados.

Tabela 3

Correlação de Spearman entre os Subtestes do WAIS-III e a Taxa de Reconhecimento das Expressões Faciais do TEPEF

\begin{tabular}{|c|c|c|c|c|c|c|c|c|c|c|c|c|}
\hline 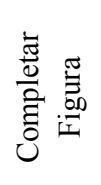 & 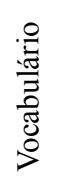 & $\begin{array}{l}0 \\
0.0 \\
: 0 \\
0 \\
0 \\
0\end{array}$ & 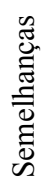 & $\frac{0}{8}$ & 䍖 & 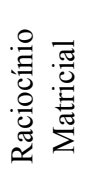 & $\frac{n}{0} \cdot \frac{0}{0,0}$ & 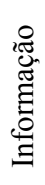 & 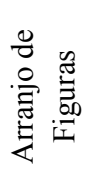 & 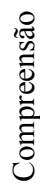 & 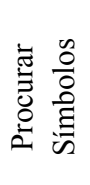 & 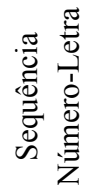 \\
\hline
\end{tabular}

\begin{tabular}{|c|c|c|c|c|c|c|c|c|c|c|c|c|c|c|}
\hline \multirow{2}{*}{ Alegria } & $0,428^{*}$ & 0,32 & $0,468^{*}$ & 0,394 & 0,307 & 0,201 & 0,333 & 0,198 & $0,517 * *$ & $0,553 * *$ & $0,414^{*}$ & 0,378 & 0,201 & $0,422^{*}$ \\
\hline & 0,037 & 0,128 & 0,021 & 0,056 & 0,144 & 0,347 & 0,112 & 0,353 & 0,010 & 0,005 & 0,044 & 0,068 & 0,346 & 0,040 \\
\hline \multirow{2}{*}{ Tristeza } & $0,503^{*}$ & $0,618 * *$ & $0,454 *$ & 0,310 & $0,512 *$ & $0,486^{*}$ & $0,539 * *$ & 0,184 & $0,432 *$ & $0,431 *$ & $0,470^{*}$ & 0,295 & $0,530 * *$ & $0,506^{*}$ \\
\hline & 0,012 & 0,001 & 0,026 & 0,140 & 0,010 & 0,016 & 0,007 & 0,388 & 0,035 & 0,035 & 0,021 & 0,162 & 0,008 & 0,012 \\
\hline \multirow{2}{*}{ Nojo } & $0,832 * *$ & $0,674 * *$ & $0,801 * *$ & $0,653 * *$ & $0,784 * *$ & $0,670 * *$ & $0,706^{* *}$ & $0,650^{* *}$ & $0,606^{* *}$ & $0,519 * *$ & $0,754 * *$ & $0,835 * *$ & $0,763 * *$ & $0,833 * *$ \\
\hline & 0,001 & 0,001 & 0,001 & 0,001 & 0,001 & 0,001 & 0,001 & 0,001 & 0,002 & 0,009 & 0,001 & 0,001 & 0,001 & 0,001 \\
\hline \multirow{2}{*}{ Surpresa } & $0,835^{* *}$ & $0,685 * *$ & $0,820 * *$ & $0,695 * *$ & $0,760 * *$ & $0,646 * *$ & $0,680 * *$ & $0,658 * *$ & $0,650 * *$ & $0,595 * *$ & $0,780 * *$ & $0,801 * *$ & $0,801 * *$ & $0,790 * *$ \\
\hline & 0,001 & 0,001 & 0,001 & 0,001 & 0,001 & 0,001 & 0,001 & 0,001 & 0,001 & 0,002 & 0,001 & 0,001 & 0,001 & 0,001 \\
\hline \multirow{2}{*}{ Medo } & 0,155 & 0,196 & 0,198 & 0,127 & 0,08 & $-0,114$ & $-0,072$ & 0,243 & 0,159 & 0,136 & 0,23 & 0,298 & 0,243 & 0,193 \\
\hline & 0,469 & 0,358 & 0,354 & 0,553 & 0,712 & 0,595 & 0,739 & 0,253 & 0,459 & 0,525 & 0,28 & 0,158 & 0,253 & 0,367 \\
\hline \multirow{2}{*}{ Raiva } & $0,847 * *$ & $0,807 * *$ & $0,828 * *$ & $0,678 * *$ & $0,720 * *$ & $0,675 * *$ & $0,758 * *$ & $0,607 * *$ & $0,623 * *$ & $0,616^{* *}$ & $0,822 * *$ & $0,676 * *$ & $0,818 * *$ & $0,715^{* *}$ \\
\hline & 0,001 & 0,001 & 0,001 & 0,001 & 0,001 & 0.001 & 0,001 & 0,002 & 0,001 & 0,001 & 0,001 & 0,001 & 0,001 & 0,001 \\
\hline
\end{tabular}

${ }^{*} p<0,05 ; * * p<0,001$.

A correlação de Spearman entre o WAIS-III e a taxa de reconhecimento das expressões faciais do TEPEF mostrou que as variáveis foram positivamente correlacionadas. As Tabelas 3 e 4 mostram essa correlação (valores na parte superior referem-se ao $r$ e na parte inferior ao índice de significância). Nojo, surpresa e raiva tiveram correlação com todos os subtestes e índices $(p<0,001)$ ao passo que medo não apresentou correlações significativas.
A expressão de alegria foi correlacionada com Completar Figura, Códigos, Informação, Arranjo de Figura, Compreensão, Armar Objeto, QI Total, ICV (Índice de Compreensão Verbal) e IVP (Índice de Velocidade de Processamento). Os dados sugerem influência de componentes verbais e da velocidade do processamento de informações visuoespaciais na percepção da face de alegria. Assim, a probabilidade de seu reconhecimento 
Ladislau, R., Guimarães, J. G. \& Souza, W. C. (2015). Percepção de Expressões Faciais Emocionais em Idosos com Doença de Alzheimer.

Tabela 4

Correlação de Spearman entre os Índices Fatoriais do WAIS-III e a Taxa de Reconhecimento das Expressões Faciais do TEPEF

\begin{tabular}{|c|c|c|c|c|c|c|c|}
\hline & $\begin{array}{l}\bar{\pi} \\
\overline{0} \overline{0} \\
\overline{0} \\
\overline{0}\end{array}$ & 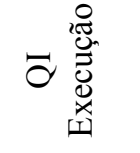 & $\begin{array}{l}\bar{\pi} \\
\stackrel{0}{0} \\
\overline{0}\end{array}$ & 己̇ & อิ & $\stackrel{0}{i}$ & $\sum$ \\
\hline Alegria & $\begin{array}{l}0,375 \\
0,071\end{array}$ & $\begin{array}{l}0,399 \\
0,054\end{array}$ & $\begin{array}{c}0,429^{*} \\
0,036\end{array}$ & $\begin{array}{c}0,431^{*} \\
0,035\end{array}$ & $\begin{array}{l}0,346 \\
0,097\end{array}$ & $\begin{array}{l}0,169 \\
0,431\end{array}$ & $\begin{array}{c}0,419 * \\
0,042\end{array}$ \\
\hline Tristeza & $\begin{array}{c}0,464^{*} \\
0,022\end{array}$ & $\begin{array}{c}0,491 * \\
0,015\end{array}$ & $\begin{array}{c}0,517 * * \\
0,010\end{array}$ & $\begin{array}{c}0,437^{*} \\
0,033\end{array}$ & $\begin{array}{c}0,518^{* *} \\
0,010\end{array}$ & $\begin{array}{c}0,468^{*} \\
0,021\end{array}$ & $\begin{array}{l}0,387 \\
0,062\end{array}$ \\
\hline Nojo & $\begin{array}{c}0,764 * * \\
0,001\end{array}$ & $\begin{array}{c}0,789 * * \\
0,001\end{array}$ & $\begin{array}{c}0,808^{* *} \\
0,001\end{array}$ & $\begin{array}{c}0,743^{* *} \\
0,001\end{array}$ & $\begin{array}{c}0,798^{* *} \\
0,001\end{array}$ & $\begin{array}{c}0,685^{* *} \\
0,001\end{array}$ & $\begin{array}{c}0,856^{* *} \\
0,001\end{array}$ \\
\hline Surpresa & $\begin{array}{c}0,791 \text { ** } \\
0,001\end{array}$ & $\begin{array}{c}0,791 * * \\
0,001\end{array}$ & $\begin{array}{c}0,822 * * \\
0,001\end{array}$ & $\begin{array}{c}0,787 * * \\
0,001\end{array}$ & $\begin{array}{c}0,782 * * \\
0,001\end{array}$ & $\begin{array}{c}0,692^{* *} \\
0,001\end{array}$ & $\begin{array}{c}0,854^{* *} \\
0,001\end{array}$ \\
\hline Medo & $\begin{array}{l}0,198 \\
0,354\end{array}$ & $\begin{array}{l}0,067 \\
0,755\end{array}$ & $\begin{array}{l}0,129 \\
0,547\end{array}$ & $\begin{array}{l}0,289 \\
0,170\end{array}$ & $\begin{array}{l}0,851 \\
0,813\end{array}$ & $\begin{array}{l}0,093 \\
0,664\end{array}$ & $\begin{array}{l}0,235 \\
0,269\end{array}$ \\
\hline Raiva & $\begin{array}{c}0,808 * * \\
0,001\end{array}$ & $\begin{array}{c}0,821 * * \\
0,001\end{array}$ & $\begin{array}{c}0,823^{* *} \\
0,001\end{array}$ & $\begin{array}{c}0,789 * * \\
0,001\end{array}$ & $\begin{array}{c}0,817 * * \\
0,001\end{array}$ & $\begin{array}{c}0,716^{* *} \\
0,001\end{array}$ & $\begin{array}{c}0,821 * * \\
0,001\end{array}$ \\
\hline
\end{tabular}

Notas. ICV = Índice de Compreensão Verbal; IOP = Índice de Organização Perceptual; IMO = Índice de Memória Operacional; IVP = Índice de Velocidade de Processamento.

$* p<0,05 ; * * p<0,001$.

aumenta com a melhora no desempenho dos subtestes e índices fatoriais citados.

A tristeza, por sua vez, foi correlacionada com Completar Figuras, Vocabulário, Códigos, Cubos, Aritmética, Raciocínio Matricial, Informação, Arranjo de Figuras, Compreensão, Sequência Número-Letra, Armar Objeto, QI Verbal, QI Execução, QI Total, ICV, IOP e IMO (Índice de Memória Operacional). Nesse caso, além da influência de aspectos verbais e visuoespaciais observados também na alegria, o reconhecimento da tristeza sofreu influência da memória operacional.

\section{Discussão}

Ao comparar os subtestes do WAIS-III com o reconhecimento de expressões emocionais, observou-se que a alegria e a tristeza tiveram menos correlações positivas com os subtestes quando comparadas com as expressões de raiva, nojo e surpresa, correlacionadas positivamente com todos os subtestes. Como sugerido no estudo de Luzzi et al. (2007), o déficit no reconhecimento de expressões faciais emocionais em idosos com DA está relacionado ao hemisfério direito do cérebro. Mais especificamente, o hemisfério direito está voltado para o processamento de emoções negativas (Perry et al., 2001). Talvez por isso, raiva e medo tenham sido menos reconhecidos. Esse é um dado em conformidade com o estudo de Kohler et al. (2005), no que se refere a uma diminuição na taxa de reconhecimento do medo. O reconhecimento da surpresa parece tornar mais provável o reconhecimento das outras expressões, pois ela foi a única expressão correlacionada positivamente com as outras cinco expressões emocionais. Além disso, ela também foi correlacionada positivamente com os subtestes do WAIS-III. Assim, à medida que o desempenho nos subtestes melhora, aumenta-se a chance de reconhecer a surpresa. O seu reconhecimento, por sua vez, favorece o reconhecimento das outras expressões.

Contudo, como a DA é uma doença degenerativa, e como a maioria dos participantes estavam acometidos em grau moderado, é possível que suas habilidades cognitivas estivessem prejudicadas como um todo. Isso certamente interferiria na percepção das expressões emocionais da face, já que a emoção é eliciada por representação mental e memória (Scherer \& Ekman, 1984).

Para o presente estudo, o WAIS-III serviu como uma bateria sensível à amostra com DA, mas com pouca especificidade, ao contrário do que propõe o estudo de Izawa, Urakami, Kojima e Ohama (2009). Eles fizeram um estudo para verificar a eficácia da versão japonesa do WAIS-III em idosos com DA e encontraram diferenças significativas para a severidade da DA nos subtestes Semelhanças, Compreensão, Aritmética, Dígitos e Sequência Número-Letras. Além disso, eles observaram que o declínio no pensamento abstrato e na solução de problemas e que a relativa preservação do IOP caracterizavam idosos com DA em estágios iniciais. 
Os dados sobre a percepção de expressão emocional facial testada pelo TEPEF foram consistentes, sugerindo que os estímulos do software podem ser uma boa medida para averiguar déficits na percepção da expressão emocional da face de idosos com DA, exceto para a face de medo. O desempenho do Grupo Controle para a percepção das expressões emocionais foi muito superior ao Grupo Experimental, confirmando a hipótese desta pesquisa.

A produção da face de medo exige o movimento de músculos muito específicos que a caracterizam genuinamente apenas na situação real de perigo (i.e. músculo triangular), conforme Darwin (1872/2000). Conforme Scherer e Ekman (1984), a presença da expressão emocional não estabelece a presença da emoção por si mesma, uma vez que ela pode ser simulada. Nesse caso, a alteração nas regiões de simulação motora e emocional em idosos com DA, evidenciada no estudo de Lee et al. (2013), pode explicar um desempenho inferior no reconhecimento da expressão emocional de medo.

O baixo desempenho no reconhecimento da expressão emocional facial em idosos com DA parece estar relacionado à progressão da demência (Lavenu \& Pasquier, 2005; Lee et al., 2013; Spoletini et al., 2008). Na progressão, há o acometimento de algumas estruturas relacionadas ao processamento das emoções (McLellan et al., 2008), como a amígdala (Wright et al., 2007), o córtex temporal anterior e o córtex frontal orbital (Mclellan et al., 2008).

Embora a presente pesquisa não tenha utilizado a análise de exame de imagem, os dados parecem estar na mesma direção, uma vez que o desempenho dos idosos com DA para o reconhecimento de faces emocionais foram prejudicados, exceto para alegria. Se fosse um prejuízo relacionado apenas às habilidades visuoespaciais, provavelmente, seriam encontrados escores baixos somente nos testes visuoespaciais, mas os dados mostraram que o Grupo Experimental obteve baixo desempenho em todas as habilidades cognitivas.

As duas faces emocionais com melhores índices de reconhecimento foram a alegria e a tristeza. A alegria obteve correlação positiva com os testes que envolvem linguagem e tempo para processar informações visuoespaciais. A tristeza obteve correlação positiva com testes que analisam as mesmas funções cognitivas da alegria, mas com o acréscimo da memória operacional (Tabelas 3 e 4).

Por outro lado, se o comprometimento fosse apenas pelo processamento emocional, a face de alegria deveria ter obtido um reconhecimento inferior no Grupo Experimental. A preservação do reconhecimento da face de alegria sugere que ainda há uma memória residual voltada para essa expressão emocional facial. Portanto, parece que os déficits encontrados na percepção estão mais relacionados à progressão da DA, que afeta tanto as habilidades visuoespaciais quanto o processamento da emoção. Coincidentemente ou não, as principais áreas relacionadas ao processamento emocional estão relacionadas à progressão da DA. Caramelli e Barbosa (2002) descrevem que a evolução da DA parte das regiões subcorticais, incluindo a formação hipocampal, e avança para regiões frontotemporais (Selkoe, 2001, citado por Sereniki \& Vital, 2008).

Contudo, estudos que mostrem comportamentalmente, por meio de testes, como essas alterações se manifestam são essenciais tanto para acompanhar a evolução do quadro, quanto para montar um programa de estimulação para os idosos e proporcionar-lhes mais qualidade de vida. Pesquisas futuras podem investir em estimulação de faces emocionais para idosos com DA. Softwares que trabalhem com expressões emocionais faciais podem contribuir para a estimulação cognitiva desses idosos e otimizar o trabalho do neuropsicólogo com feedbacks constantes e registros individuais e longitudinais do paciente em reabilitação.

O presente estudo teve resultados importantes na avaliação de cinco expressões emocionais faciais e na diferenciação das amostras por meio dos testes aplicados. Os limites ficaram no pareamento por idade e sexo, na expressão de medo e na especificidade dos testes escolhidos. A discrepância entre os grupos controle e experimental quanto à idade deveu-se, provavelmente, à forma com que a amostra do Grupo Controle foi selecionada, pelo método bola-de-neve, que pressupõe a indicação de pessoas próximas. Assim, havia uma tendência da faixa etária ser semelhante neste grupo. Já no caso do Grupo Experimental, esse método não foi utilizado. Nesse contexto, houve dificuldade pela análise de prontuários, alguns dos quais estavam desatualizados em relação ao CDR, o que comprometeu o tamanho da amostra. Além disso, a amostra foi pequena, dificultando a generalização dos resultados.

Embora o WAIS-III seja validado e padronizado para a população brasileira, os testes sugeridos por Nitrini et al. (2005) devem ser considerados. Eles propuseram um consenso relacionado à avaliação de cada função cognitiva de idosos com DA. Assim, a sugestão se torna importante, pois oferece informações qualitativas da capacidade cognitiva dos idosos e contribui para uma padronização na avaliação clínica. Além disso, validar esses testes será de grande valia para as pesquisas e para as avaliações neuropsicológicas, em especial as relacionadas à DA.

Conclui-se, neste artigo, que há prejuízo na percepção de faces emocionais negativas para o Grupo Experimental. As implicações da dificuldade de reconhecimento das faces emocionais negativas envolvem prejuízos nas interrelações e na autoproteção. Isso porque as emoções de medo e de raiva, por exemplo, podem sinalizar situações de perigo iminente. Não percebê-los poderia comprometer a integridade do idoso. Ressalta-se, pois, a necessidade de acompanhar os longevos continuamente.

\section{Referências}

Abrisqueta-Gomez, J., Bueno, O. F. A., Oliveira, M. G. M., \& Bertolucci, P. H. F. (2002). Recognition memory for emotional pictures in Alzheimer's patients. Acta Neurologica Scandinavica, 105, 51-54.

Bentley, P., Driver, J., \& Dolan, R. J. (2008). Cholinesterase inhibition modulates visual and attentional brain responses in Alzheimer's disease and health. Brain, 131, 409-424. 
Blair, R. J., \& Cipolotti, L. (2000). Impaired social response reversal: A case of acquired sociopathy'. Brain, 123(6), $1122-1141$.

Brucki, S. M., Nitrini, R., Caramelli, P., Bertolucci, P. H., Ivan, H., \& Okamoto, I. H. (2003). Sugestões para o uso do Mini-Exame do Estado Mental no Brasil. Arquivos de Neuropsiquiatria, 61(3-B), 777-781.

Busigny, T., \& Rossion, B. (2010). Acquired prosopagnosia abolishes the face inversion effect. Cortex, 46, 965-981.

Burnham, H., \& Hogervorst, E. (2004). Recognition of facial expressions of emotion by patients with dementia of the Alzheimer type. Dementia and Geriatric Cognitive Disorders, 18(1), 75-79.

Caramelli, C., \& Barbosa, M. T. (2002). Como diagnosticar as quatro causas mais freqüentes de demência? Revista Brasileira de Psiquiatria, 24(Supl. 1), 7-10.

Celone, K. A., Calhoun, V. D., Dickerson, B. C., Atri, A., Chua, E. F., Miller, S. L., ...Sperling, R. A. (2006). Alterations in memory networks in mild cognitive impairment and Alzheimer's disease: An independent component analysis. The Journal of Neuroscience, 26(40), 10222-10231.

Choleris, E., Kavaliers, M., \& Pfaff, D. (2008). Brain mechanisms theoretically underlying extremes of social behaviors: The best and worst. Hormones and Social Behavior, 13-25.

Darwin, C. (2000). A expressão das emoções no homem e nos animais. São Paulo, SP: Companhia das Letras. (Original publicado em 1872)

De Souza, W. C., Feitosa, M. A. G., Eifuku, S., Tamura, R., \& Ono, T. (2008). Face perception in its neurobiological and social context. Psychology \& Neuroscience, 1, 15-20.

Drapeau, J., Gosselin, N., Gagnon, L., Peretz, I., \& Lorrain, D. (2009). Emotional recognition from face, voice, and music in dementia of the Alzheimer type. Annals of the New York Academy of Sciences, 1169, 342-345.

Ekman, P., \& Friesen, W. V. (1971). Constants across cultures in the face and emotion. Journal of Personality and Social Psychology, 17(2), 124-129.

Ekman, P., \& Friesen, W. V. (1978). Facial action coding system. Palo Alto, CA: Consulting Psychologists Press.

Farah, M. J., \& Feinberg, T. E. (2005). Patient-based approaches to cognitive neuroscience ( $2^{\text {nd }} \mathrm{ed}$.). London: Massachusetts Institute of Technology Press.

Folstein, M. F., Folstein, S. E., \& McHugh, P. R. (1975). "Minimental state": A practical method for grading the cognitive state of patients for the clinician. Journal of Psychiatric Research, 12(3), 189-198.

Forlenza, O. V., \& Gattaz, W. F. (1998). Influência de mecanismos colinérgicos nos processos neurodegenerativos relacionados à formação de amilóide e à fosforilação da proteína tau. Revista de Psiquiatria Clínica, 25(3), 114-117.

Guaita, A., Malnati, M., Vaccaro, R., Pezzati, R., Marcionetti, J., Vitali, S. F., \& Colombo, M. (2009). Impaired facial emotion recognition and preserved reactivity to facial expressions in people with severe dementia. Archives of Gerontology and Geriatrics, 49, 135-146.

Grady, C. L., Furey, M. L., Pietrini, P., Horwitz, B., \& Rapoport, S. I. (2001). Altered brain functional connectivity and impaired short-term memory in Alzheimer's disease. Brain, 124 (Pt. 4), 739-756.

Hargrave, R., Maddock, R. J., \& Stone, V. (2002). Impaired recognition of facial expressions of emotion in Alzheimer's disease. Journal of Neuropsychiatry Clinical Neuroscience, 14(1), 64-71.
Haxby, J. V., Hoffman, E. A., \& Gobbini, M. I. (2002). Human neural system for face recognition and social communication. Society of Biological Psychiatry, 51, 59-67.

Izawa, Y., Urakami, K., Kojima, T., \& Ohama, E. (2009). Wechsler Adult Intelligence Scale, $3^{\text {rd }}$ Edition (WAIS-III): Usefulness in the early detection of Alzheimer's disease. Acta Medica, 52, 11-20.

Kipps, C. M., Duggins, A. J., McCusker, E. A., \& Calder, A. J. (2007). Disgust and happiness recognition correlate with anteroventral insula and amydala volume respectively in preclinical Huntington's disease. Journal of Cognitive Neuroscience, 19, 1206-1217.

Kohler, C. G., Anselmo-Gallagher, G., Bilker, W., Karlawish, J., Gur, R. E., \& Clark, C. M., (2005). Emotion-discrimination deficits in mild Alzheimer disease. The American Journal of Geriatric Psychiatry, 13, 926-933.

Ladislau, R. L. (2010). Percepção de expressões faciais emocionais em idosos com doença de Alzheimer (Dissertação de mestrado, Universidade de Brasília, DF, Brasil).

Lavenu, I., \& Pasquier, F. (2005). Perception of emotion on faces in frontotemporal dementia and Alzheimer's disease: A longitudinal study. Dementia and Geriatric Cognitive Disorders, 19(1), 37-41.

Lee, T. M. C., Sun, D., Leung, M. K., Chu, L. W., \& Keysers, C. (2013). Neural activities during affective processing in people with Alzheimer's disease. Neurobiology of Aging, 34(3), 706-715.

Luzzi, S., Piccirilli, M., \& Provinciali, L. (2007). Perception of emotions on happy/sad chimeric faces in Alzheimer disease: Relationship with cognitive functions. Alzheimer Disease and Associated Disorders, 21(2), 130-135.

McLellan, T., Johnston, L., Dalrymple-Alford, J., \& Porter, R. (2008). The recognition of facial expressions of emotion in Alzheimer's disease: A review of findings. Acta Neuropsychiatrica, 20, 236-250.

Morris, J. C. (1997). Clinical Dementia Rating: A reliable and valid diagnostic and staging measure for Dementia of the Alzheimer type. International Psychogeriatrics, 9(Suppl. 1), S173-S176

Nitrini, R., Caramelli, P., Bottino, C. M. C., Damasceno, B. P., Brucki, S. M. D., \& Anghinah, R. (2005). Diagnóstico de doença de Alzheimer no Brasil: Avaliação cognitiva e funcional - Recomendações do Departamento Científico de Neurologia Cognitiva e do Envelhecimento da Academia Brasileira de Neurologia. Arquivos de Neuropsiquiatria, 63(3-A), 720-727.

O’Brien, H. L., Tetewsky, S. J., Avery, L. M., Cushman, L. A., Makous, W., \& Duffy, C. J. (2001). Visual mechanisms of spatial disorientation in Alzheimer's disease. Cerebral Cortex 11, 1083-1092.

Perry, R. J., Rosen, H. R., Kramer, J. H., Beer, J. S., Levenson, R. L., \& Miller, B. L. (2001). Hemispheric dominance for emotions, empathy and social behavior: Evidence from right and left handers with frontotemporal dementia. Neurocase, 7, 145-160.

Ramon, M., Busigny, T., \& Rossion, B. (2010). Impaired holistic processing of unfamiliar individual faces in acquires prosopagnosia. Neurospychologia, 48, 933-944.

Sato, W., Kubota, Y., Okada, T., Murai, T., Yoshikawa, S., \& Sengoku, A. (2002). Seeing happy emotion in fearful and angry faces: Qualitative analysis of facial expression recognition in a bilateral amygdala-damaged patient. Cortex 38(5), 727-742. 
Scherer, K., \& Ekman, P. (1984). Approaches to emotion. Hillsdale, NJ: Lawrence Erlbaum.

Schultz, R. (2003). Memória emocional, volume do corpo amigdalóide e doença de Alzheimer (Tese de doutorado, Universidade Federal de São Paulo, SP, Brasil).

Sereniki, A., \& Vital, M. A. B. F. (2008). A doença de Alzheimer: Aspectos fisiopatológicos e farmacológicos. Revista de Psiquiatria do Rio Grande do Sul, 30(Supl. 1).

Spikman, J. M., Milders, M. V., Visser-Keizer, A. C., WesterhofEvers, H. J., Herben-Dekker, M., \& van der Naalt, J. (2013). Deficit en facial emotion recognition indicate behavioral changes and self-awareness after moderate to severe traumatic brain injury. PLoS ONE, 8(6), e65581.

Spoletini, I., Marra, C., Di Iulio, F., Gianni, W., Sancesario, G., Giubilei, F., ...Spalletta, G. (2008). Facial emotion recognition déficit in amnestic mild cognitive impairment and Alzheimer Disease. American Journal of Geriatric Psychiatry, 16, 389-398.

Tessitore, A., Hariri, A. R., Fera, F., Smith, W. G., Chase, T. N., Hyde, T. M., ...Mattay, V. S. (2002). Dopamine modulates the response of the human amygdala: A study in Parkinson's disease. Journal of Neuroscience, 22(20), 9099-9103.

Wechsler, D. (2004). WAIS-III: Escala de Inteligência Wechsler para Adultos: Manual/David Wechsler (E. do Nascimento, Adapt. brasileira). São Paulo, SP: Casa do Psicólogo.

Weigelt, S., Koldewyn, K., \& Kanwisher, N. (2011). Face identity recognition in autism spectrum disorders: A review of behavioral studies. Neuroscience and Biobehavioral Reviews, 1061-1082.

Werheid, K., \& Clare, L. (2007). Are faces special in Alzheimer's disease? Cognitive conceptualisation, neural correlates, and diagnostic relevance of impaired memory for faces and names. Cortex, 43(7), 898-906.

Wright, C. I., Dickerson, B. C., Feczko, E., Negeira, A., \& Williams, D. (2007). A functional magnetic resonance imaging study of amygdala responses to human faces in aging and mild Alzheimer's disease. Biological Psychiatry, 62(12), 1388-1395.

Yesavage, J. A., Brink, T. L., Rose, T. L., Lum, O., Huang, V., Adey, M., \& Leirer, V. O. (1982). Development and validation of a Geriatric Depression Screening Scale: A preliminary report. Journal of Psychiatric Research, 17, 37-49.

Yoshimura, N., Kawamura, M., Masaoka, Y., \& Homma, I. (2005). The amygdala of patients with Parkinson's disease is silent in response to fearful facial expressions. Neuroscience, 131, 523-534. 